\title{
Nano-microcapsule basic fibroblast growth factor combined with hypoxia-inducible factor-1 improves random skin flap survival in rats
}

\author{
GUANG-JUN CHEN, YI-HENG CHEN, XIA-QING YANG and ZHI-JIE LI \\ Department of Hand and Plastic Surgery, The 2nd Affiliated Hospital of Wenzhou Medical University, \\ Wenzhou, Zhejiang 325027, P.R. China
}

Received March 22, 2015; Accepted October 29, 2015

DOI: $10.3892 / \mathrm{mmr} .2015 .4699$

\begin{abstract}
The present study aimed to investigate the effect of nano-microcapsule-basic fibroblast growth factor (bFGF) combined with hypoxia-inducible factor-1 (HIF-1) on the random skin flap survival of rats. Male Sprague-Dawley rats were used to establish the McFarlane flap model and subsequently, all model rats were randomly divided into four groups: Control, bFGF, HIF-1 and bFGF combined with HIF-1. The model rats were treated with $2.5 \mu \mathrm{g} /$ day bFGF and $1.0 \mu \mathrm{g} /$ day HIF-1 for 5 days by intraperitoneal injection. On day 5 following treatment, the boundaries between necrotic and surviving regions were significantly inhibited by bFGF combined with HIF-1. bFGF combined with HIF-1 inhibited oxidative stresses and inflammatory factors in random skin flap survival of rats. bFGF combined with HIF-1 also activated the protein expression levels of cyclooxygenase (COX)-2 and vascular endothelial growth factor (VEGF) in the random skin flap survival of rats. In conclusion, nano-microcapsule bFGF combined with HIF-1 prevented random skin flap survival in rats through antioxidative, anti-inflammatory and activation of the protein expression levels of COX-2 and VEGF.
\end{abstract}

\section{Introduction}

Random flap is widely used in the repair and reconstruction of tissue defect due to its convenience and flexibility (1). However, since no known blood vessels are found in the random flap, the distal end is prone to necrosis, which greatly limits its application in clinical practice (2). The ischemic injury mechanism of random flap remains to be fully elucidated (3).

Correspondence to: Mr. Zhi-Jie Li, Department of Hand and Plastic Surgery, The 2nd Affiliated Hospital of Wenzhou Medical University, 109 Xueyuan Road, Wenzhou, Zhejiang 325027, P.R. China

E-mail: lizjzjli@163.com

Key words: bFGF, HIF-1, random skin flap survival, anti-oxidative, anti-inflammatory, COX-2, VEGF
Several growth factors, including basic fibroblast growth factor (bFGF), are used to improve the survival rate of flap and are applied to the research of promoting angiogenesis (4). bFGF is susceptible to proteolytic degradation in vivo, which is destroyed and degraded rapidly in the body due to its short half-life, and the biological effect of partial use remains to be elucidated (5). Using a microsphere system to control the local delivery of growth factor may be an effective method for solving the above problem (6).

Hypoxic inducible factor (HIF)- 1 is a transcription factor widely present in the body of mammals and humans under hypoxic conditions, which is highly sensitive to the concentration of oxygen in the environment (7). With a prolonged hypoxic duration, the quantity of HIF-1 $\alpha$ protein also increases, and once hypoxia is improved, HIF-1 $\alpha$ levels decline rapidly (8). Previous studies have shown that the gene expression of HIF in the ischemic area is upregulated, suggesting that the gene expression of HIF may be associated with local hypoxia following ischemia, endothelial progenitor cell (EPC) amplification and improvement of ischemia by promoting angiogenesis $(9,10)$. The present study aimed to investigate whether bFGF combined with HIF-1 improved random skin flap survival of rats.

\section{Materials and methods}

Materials. bFGF, HIF-1, superoxide dismutase (SOD) and malondialdehyde (MDA) were obtained from Nanjing KeyGen Biotech. Co., Ltd., (Nanjing, China). Anti-cyclooxygenase (COX)-2, anti-vascular endothelial growth factor (VEGF) and anti- $\beta$-actin antibodies were obtained from Bioworld Technology (Nanjing, China).

Animals. Male Sprague-Dawley rats (weighing, 260-300 g) were obtained from Laboratory Animals of Wenzhou Medical University (Wenzhou, China) and were treated in accordance with the Guide for the Care and Use of Laboratory Animals of The 2nd Affiliated Hospital of Wenzhou Medical University (Wenzhou, China). The rats were housed in separate clean cages at a temperature of $20-24^{\circ} \mathrm{C}$ under $12 \mathrm{~h}$ light/dark cycles, with chow pellets and tap water available ad libitum. 
Flap model and experimental design. Random dorsal skin flaps were elevated using the previously described model (11). The rats were anesthetized with sodium pentobarbitone $(40 \mathrm{mg} / \mathrm{kg}$; Sigma-Aldrich, St. Louis, MO, USA) by intraperitoneal injections. The dorsal skin was shaved and the rats were placed into the prone position by securing their limbs with adhesive tape. Povidone iodine solution (Shandong Lierkang Disinfection Technology Co., Ltd., Shandong, China) was used to disinfect the skin and all surgical procedures were performed under sterile conditions. Full-thickness rectangular skin incisions were made on the dorsum of each animal, and were immediately sutured with interrupted 4-0 nylon sutures (Fig. 1).

All rats were randomly divided into four groups (n=10/group): (i) Control; (ii) Nano-microcapsule-bFGF; (iii) Nano-microcapsule-HIF-1; and (iv) bFGF combined with HIF-1 (combined). In the control group, the model rats were treated with intraperitoneal injections of physiological saline $(0.1 \mathrm{ml} / 100 \mathrm{~g})$. The bFGF group rats were treated with $2.5 \mu \mathrm{g}$ /day bFGF for 5 days by intraperitoneal injection and the HIF-1 group rats were treated with $1.0 \mu \mathrm{g} /$ day HIF-1 for 5 days by intraperitoneal injection. In the combined treatment group, the rats were treated with bFGF and HIF-1 at the same concentration as single treatment groups for 5 days by intraperitoneal injections.

Assessment of the expression levels of bFGF, HIF-1 MDA, $S O D, T N F-\alpha$ and $I L-1 \beta$. Following combined treatment, blood sample were acquired from the vein of flap tissues at room temperature. The serum levels of bFGF, HIF-1, MDA, SOD, TNF- $\alpha$ and IL- $1 \beta$ were analyzed using enzyme linked immunosorbent assay kits, according to the manufacturer's protocols (KeyGen Biotech. Co., Ltd,. Nanjing, China).

Assessment of survival areas. Following combined treatment, the flap tissues were biopsied for histological assessment and immunohistochemical staining of VEGF. The flaps and surviving areas were measured and images were captured with superimposition of photographs on graph paper. The images were exposed in a ChemiDoc MP Imaging System (Bio-Rad Laboratories, Inc., Hercules, CA, USA) The computational formula was calculated as follows: Extent of viable area $\mathrm{x} 100$ / total area.

Western blot assay for COX-2 and VEGF. Following combined treatment, the total cellular protein was extracted from the flap tissues. The flap tissues were homogenized using a radioimmunoprecipitation lysis buffer (Nanjing Sunshine Biotechnology, Co., Ltd., Nanjing, China). The homogenized tissue samples were centrifuged at $12,000 \mathrm{x}$ g for $10 \mathrm{~min}$ at $4^{\circ} \mathrm{C}$ and the supernatant liquor was subsequently absorbed into the new tube. The concentration of the total cellular protein was quantified using a BCA Protein Assay (Pierce Biotechnology Inc., Rockford, IL, USA). Equal quantities of protein $(50 \mu \mathrm{g})$ were separated by electrophoresis using 12\% SDS-PAGE gels (Beyotime Institute of Biotechnology, Haimen, China) and were transferred onto polyvinylidene fluoride membranes (EMD Millipore, Billerica, MA, USA). Following protein transfer, the membranes were blocked with $5 \%$ non-fat milk and incubated with the corresponding primary antibodies, anti-COX-2 (1:1,000 dilution; cat. no. sc-514489, Santa Cruz Biotechnology, Inc., Santa Cruz,
CA, USA), anti-VEGF (1:1,000; cat. no. sc-48835; Santa Cruz Biotechnology, Inc.) and rabbit anti-mouse anti- $\beta$-actin (1:500 dilution; cat. no. AA132; Beyotime Institute of Biotechnology, Haimen, China) overnight at $4^{\circ} \mathrm{C}$. The membranes were incubated with secondary antibodies (1:5,000; C520011-0100; Sangon Biotech Co., Ltd., Shanghai, China) for $2-3 \mathrm{~h}$ at $37^{\circ} \mathrm{C}$ and were subsequently incubated with chemiluminescence detection reagent (P0203, Beyotime Institute of Biotechnology). Images were captured using Image-Pro Plus software, version 6.0 (Media Cybernetics, Rockville, MD, USA).

Statistical analysis. The data are expressed as the mean \pm standard deviation and were analyzed by one-way analysis of variance using SPSS 17.0 statistical software (SPSS, Inc., Chicago, IL, USA). P $<0.05$ was considered to indicate a statistically significant difference.

\section{Results}

bFGF expression. On day 5 following treatment, the expression of bFGF was assessed. As compared with control, the expression of bFGF was significantly advanced in bFGF group (Fig. 2). Additionally, the expression of bFGF in the HIF-1 group was similar to that of the control group (Fig. 2). In the combined treatment group, the expression of bFGF was similar to that of bFGF group (Fig. 2).

HIF-1 expression. On day 5 following treatment, the expression of HIF-1 was detected. The expression of HIF-1 in the bFGF group was similar to that of the control group, and significantly lower compared with that of the HIF-1 group (Fig. 3). The expression of HIF-1 in the HIF-1 and combined treatment groups exhibited similar changes and was significantly higher compared with the control or bFGF groups (Fig. 3).

Combined treatment improves flap survival. On day 5 following treatment, whether the combination treatment improved flap survival was assessed. As shown in Fig. 4, the boundaries between necrotic and surviving regions were highest in the control group. In the bFGF and the HIF-1 groups, the boundaries between necrotic and surviving regions were significantly inhibited compared with those of the control group (Fig. 4). These necrotic and surviving regions in the combined treatment group were lower compared with those of the bFGF or HIF-1 groups, and the difference were statistically significant (Fig. 4).

Combined treatment affects the levels of MDA and SOD. On day 5 following treatment, whether combined treatment affected the MDA and SOD levels was assessed. Firstly, bFGF treatment significantly reduced and increased the levels of MDA and SOD, respectively, compared with those of control group (Fig. 5). The HIF-1 group exhibited similar changes to the control group (Fig. 5). Finally, these oxidative stresses were significantly receded by combined treatment compared with those of the bFGF or HIF-1 groups (Fig. 5).

Combined treatment affects the levels of TNF- $\alpha$ and $I L-1 \beta$. On day 5 following treatment, whether combined treatment affected the MDA and SOD levels was determined. The TNF- $\alpha$ and IL-1 $\beta$ levels in the bFGF or HIF-1 groups were significantly 


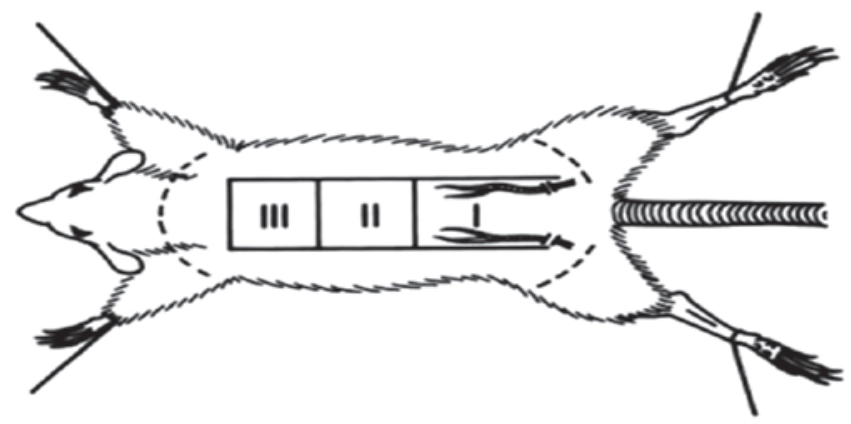

Figure 1. Schematic of the McFarlane flap model.

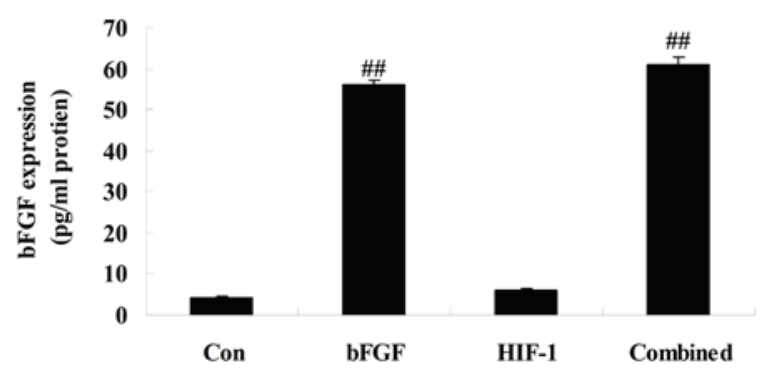

Figure 2. Expression of bFGF under the various experimental conditions. The expression levels of bFGF was assessed in the Con, bFGF, HIF-1 and combined (bFGF and HIF-1) group ${ }^{\# \# ~} \mathrm{P}<0.01$ vs. the Con group without propofol treatment). Con, control; bFGF, basic fibroblast growth factor; HIF-1, hypoxia-inducible factor-1.

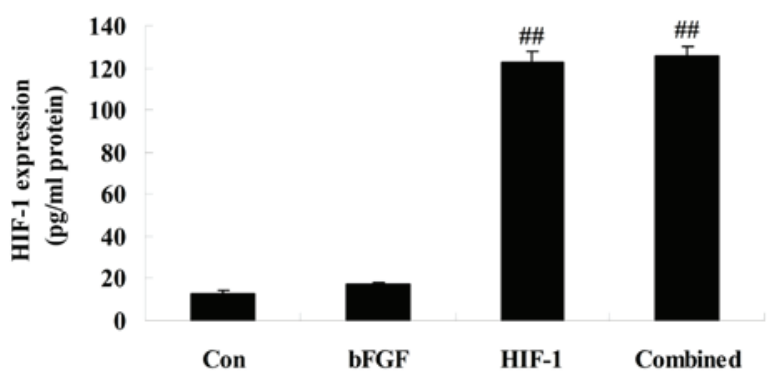

Figure 3. Expression of HIF-1 under the various experimental conditions. The expression levels of HIF-1 was assessed in the Con, bFGF, HIF-1 and combined (bFGF and HIF-1) group ${ }^{\# \# ~} \mathrm{P}<0.01$ vs. the Con group without propofol treatment). Con, control; bFGF, basic fibroblast growth factor; HIF-1, hypoxia-inducible factor-1.

weakened compared with those of the control group (Fig. 6). However, these inflammatory factors were significantly weakened in the combined treatment group compared with those of the bFGF or HIF-1 groups (Fig. 6).

Combined treatment affects the expression of COX-2. To check whether combined treatment affects the expression of COX-2 following 5 days of treatment, the protein expression of COX-2 was analyzed by western blotting. A significant difference in the protein expression of COX-2 was observed between the bFGF and HIF-1 groups (Fig. 7). When compared with that of the bFGF or HIF-1 groups, the protein expression of COX-2 in the combined treatment group was significantly increased (Fig. 7).

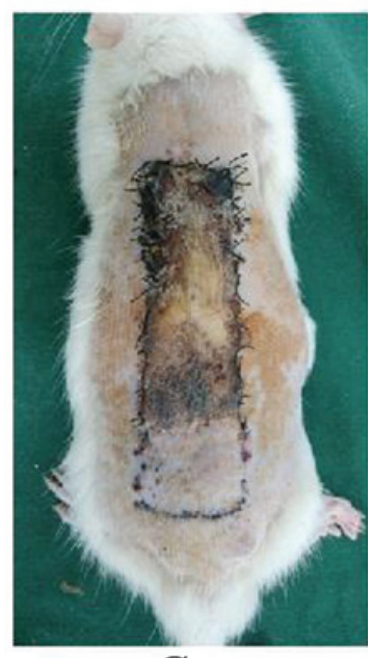

Con

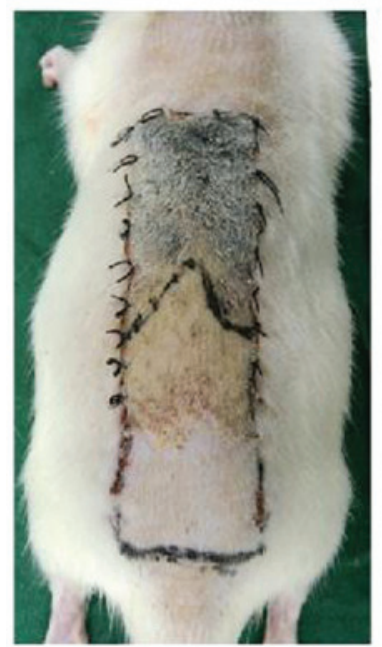

HIF-1

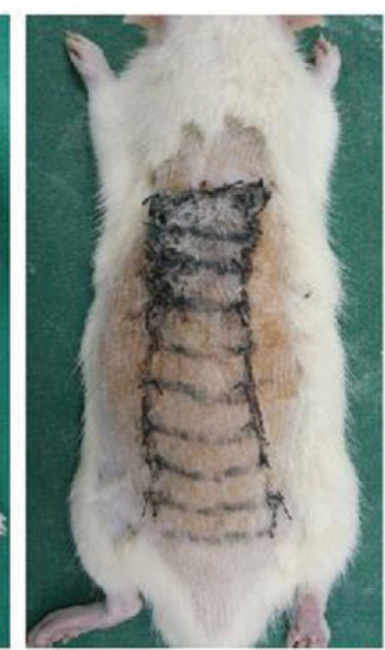

bFGF

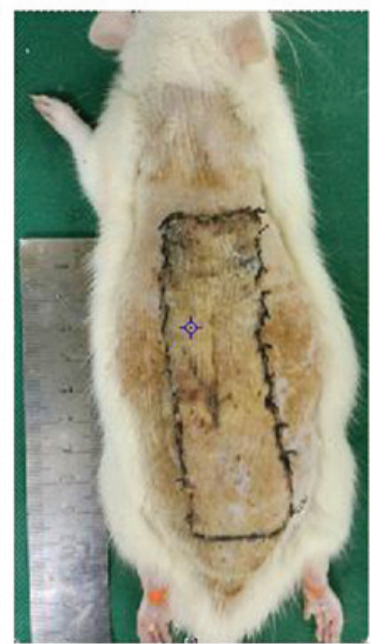

Combined
Figure 4. Combined treatment improves flap survival compared with bFGF or HIF-1 in isolation. Con, rats were treated with intraperitoneal injections of physiological saline $(0.1 \mathrm{ml} / 100 \mathrm{~g})$; bFGF, rats were treated with $2.5 \mu \mathrm{g} / \mathrm{day}$ bFGF for 5 days by intraperitoneal injection; HIF-1, rats were treated with $1.0 \mu \mathrm{g}$ /day HIF-1 for 5 days by intraperitoneal injection; combined, rats were treated with bFGF and HIF-1 at the same concentration as the single treatment groups for 5 days by intraperitoneal injections. bFGF, basic $g$ rowth fibroblast factor; HIF, hypoxia-inducible factor-1.

Combined treatment affects the expression of VEGF. The present study next determined whether combined treatment affected the expression of COX-2 following 5 days of treatment. As shown in Fig. 7, the protein expression of COX-2 was significantly promoted in the bFGF and HIF-1 groups, as compared with that of the control group (Fig. 8). Notably, the combined treatment significantly increased the protein expression of COX-2, as compared with that of the control group (Fig. 8).

\section{Discussion}

bFGF is composed of 155 amino acids and has a high affinity with dextran, in addition to heparan sulfate proteoglycans. bFGF has no signal peptide, however, exists in a paracrine or autocrine form outside the cell, the explanation of how remains 
A

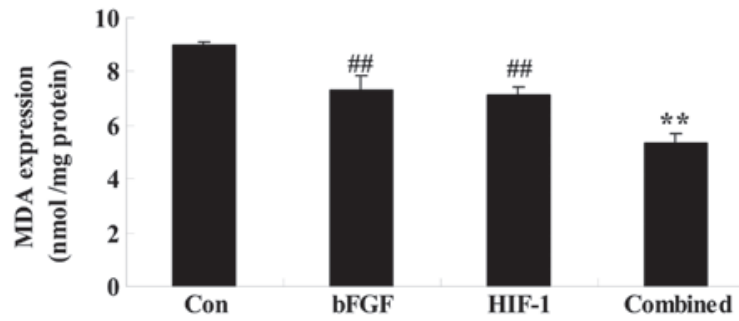

B 20

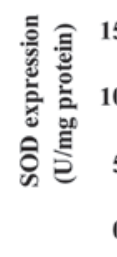

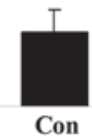
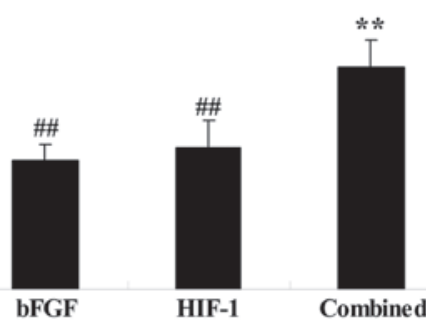

Figure 5. Expression of MDA and SOD under the various experimental conditions. Combined treatment with bFGF and HIF-1 affected the expression levels of (A) MDA and (B) SOD $\left({ }^{\#} \mathrm{P}<0.01\right.$ vs. the control group without propofol treatment; ${ }^{* *} \mathrm{P}<0.01$ vs. the bFGF or HIF group). Con, control; bFGF, basic growth fibroblast factor; HIF, hypoxia-inducible factor-1; MDA, malondialdehyde; SOD, superoxide dismutase.

A

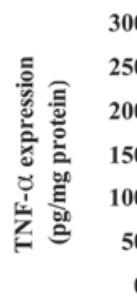

300

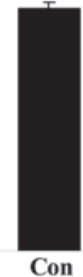

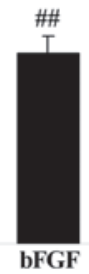
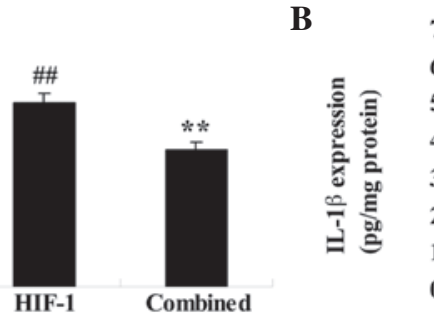
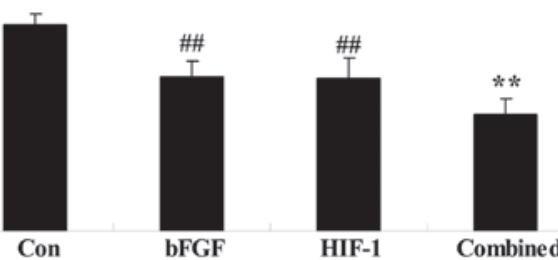

Figure 6. Expression of TNF- $\alpha$ and IL-1 $\beta$ under the various experimental conditions. Combined treatment with bFGF and HIF-1 affected the expression levels of (A) TNF- $\alpha$ and (B) IL-1 $\beta\left({ }^{* \#} \mathrm{P}<0.01\right.$ vs. the control group without propofol treatment; ${ }^{* * *} \mathrm{P}<0.01$ vs. the bFGF or HIF group). Con, control; bFGF, basic growth fibroblast factor; HIF, hypoxia-inducible factor-1; TNF- $\alpha$, tumor necrosis factor- $\alpha$; IL-1 $\beta$, interleukin- $1 \beta$.

A

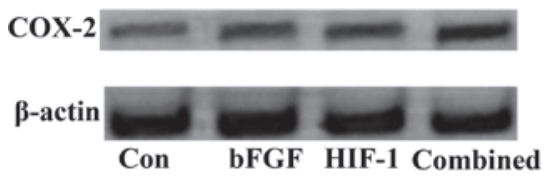

B

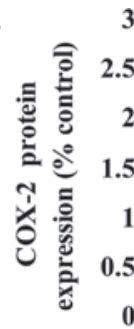

(20)

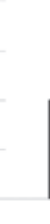

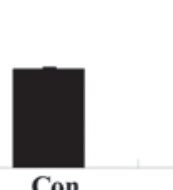

Con

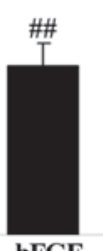

bFGF

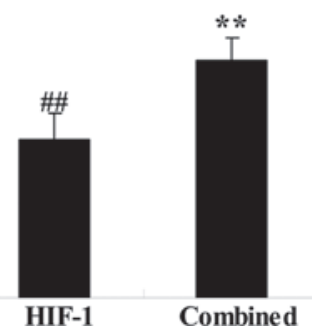

Figure 7. Expression of COX-2 under various experimental conditions. The combined treatment affected the expression levels of COX-2. (A) The results of western blot analysis revealed that the combined treatment affected the expression of COX-2 and (B) shows the quantification of this data. $\beta$-actin was used as a loading control ("\# $\mathrm{P}<0.01$ vs. the control group without propofol treatment; ${ }^{* *} \mathrm{P}<0.01$ vs. the bFGF or HIF group). Con, control; bFGF, basic growth fibroblast factor; HIF, hypoxia-inducible factor-1; COX-2, cyclo-oxygenase-2.

A

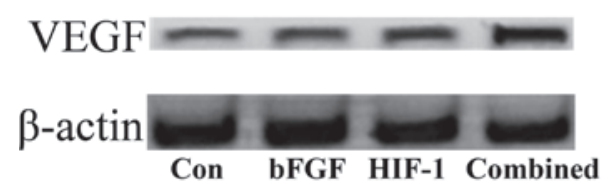

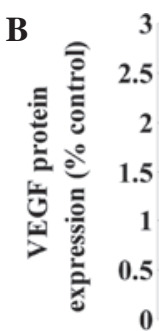

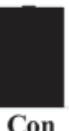

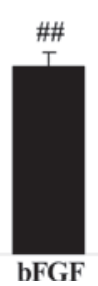

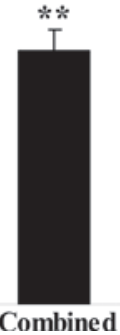

Figure 8. Expression levels of VEGF under various experimental conditions. The combined treatment affected the expression levels of VEGF. (A) The results of western blot analysis revealed that the combined treatment affected the expression of COX-2 and (B) shows the quantification of this data. $\beta$-actin was used as a loading control $\left({ }^{\# \#} \mathrm{P}<0.01\right.$ vs. the control group without propofol treatment; ${ }^{* *} \mathrm{P}<0.01$ vs. the bFGF or HIF group). Con, control; bFGF, basic growth fibroblast factor; HIF, hypoxia-inducible factor-1; VEGF, vascular endothelial growth factor.

to be elucidated (12). Due to the increase of bFGF in wounded tissue, the secretion of bFGF is assumed to be based on cell damage and exocytosis (13). However, domestic and foreign studies revealed that a large number of bFGFs are secreted following the effect of mitogens, including phorbol myristate acetate (14). In the present study, the effect of nano-microcapsule
bFGF combined with HIF-1 on the random skin flap survival of rats was investigated. Fayazzadeh et al (15) reported that recombinant human bFGF and erythropoietin protected skin flap ischemic necrosis. Therefore, nano-microcapsule bFGF combined with HIF-1 may be a beneficial pattern for random skin flap survival. 
HIF-1 $\alpha$ protein is markedly unstable in normoxic condition, likely to be decomposed by the ubiquitin-proteasome system, of which the half-life is $>5 \mathrm{~min}$. Therefore HIF-1 $\alpha$ protein is difficult to detect under normoxic conditions (16). The stability of HIF1 $\alpha$ under normoxic conditions is not enough to activate HIF-1, and only inhibiting the degradation of ubiquitin and the proteasome is not enough to produce the transcriptional activity of HIF-1, therefore, it should be transformed into exogenous HIF-1 $\alpha$, to promote its combination with structural ARNT in vivo to generate active HIF-1. By transfecting exogenous HIF-1 $\alpha$ into peripheral HIF-1 $\alpha$ EPC of humans, the previous study aimed to observe its effect on the EPC directed differentiation and vascular activity in normoxic condition (17). In the present study, nano-microcapsule bFGF combined with HIF-1 significantly weakened oxidative stresses and inflammatory factors in the random skin flap survival of rats. A previous study has demonstrated that bFGF reduces bisphosphonate-induced oxidative in oral epithelium of rat (18). Wang et al also demonstrated bFGF ischemic oxidative injury through the upregulation of the ERK1/2 pathways (19). Tasso et al (20) reported that the inflammatory response on the ability of mesenchymal stem cells to activate endogenous regenerative mechanisms is reduced by bFGF. Rodriguez-Miguelez et al (21) indicated that HIF-1 $\alpha$ modulates inflammation and the expression of VEGF. However, the detailed mechanisms on how nano-microcapsule bFGF combined with HIF-1 influences oxidative stresses and inflammatory factors in random skin flap survival of rats remain to be elucidated, and further clarification is required in a future study.

COX is a rate-limiting enzyme synthesized by prostaglandin, and there are three known isomers, COX-1, COX-2 and COX-3, in which the expression of COX- is induced, and it is undetectable in most normal tissues, however the expression is significantly upregulated under various stimuli, including cytokines, growth factors, oncogenes and tumor-promoting agents, involved in inflammation, angiogenesis, cell proliferation and differentiation processes (22). The expression of COX-2 may promote an angiogenesis effect via VEGF (23). In the present study, nano-microcapsule bFGF combined with HIF-1 increased the protein expression of COX-2 in the random skin flap survival of rats. Erdem et al (24) showed that the association between bFGF and astrocyte elevated gene-1 increased COX-2 markers with prognostic factors in prostate carcinomas. The results of Seo et al (25) suggested that activation of HIF-1 $\alpha$ upregulates COX-2 and matrix metalloproteinase-9, and prevents acinar cell death. However, the specific mechanisms of nano-microcapsule bFGF combined with HIF-1 on the expression of COX-2 remains unclear in the random skin flap survival of rats, and further clarification is required in a future study.

VEGF is also known as vascular permeability factor or angiogenic factor, which is a glycoprotein that can specifically act on the surface receptors in vascular endothelial cells, with several biological effects, including pro-vascular endothelial cell proliferation and migration, angiogenesis, vascular endothelial cell life extension and vascular permeability increasing $(26,27)$. The present study revealed that nano-microcapsule bFGF combined with HIF-1 also increased the protein expression of VEGF in the random skin flap survival of rats. Chen et al (28) indicated that inhibiting HIF-1 reduced VEGF in human colorectal cancer cells. Tzeng et al (29) also demonstrated that bFGF promotes endothelial progenitor cells and induces VEGF expression in chondrosarcoma cells. These results implied that the effect of nano-microcapsule bFGF combined with HIF-1 on random skin flap survival was associated with the expression of VEGF, and further clarification is required in a future study.

In conclusion, nano-microcapsule bFGF combined with HIF-1 prevents random skin flap survival in rats. The present study, therefore, confirmed that nano-microcapsule bFGF combined with HIF-1 may be a protective effect in terms of oxidative stresses and inflammatory activity and activates COX-2 and VEGF signal channel.

\section{Acknowledgements}

The present study was supported by the Zhejiang Provincial Medical Science Research Foundation (no. 2009A146) and the Wenzhou Science \& Technology Foundation of China (no. 2013S0430).

\section{References}

1. Cao B, Wang L, Lin D, Cai L and Gao W: Effects of lidocaine on random skin flap survival in rats. Dermatol Surg 41: 53-58, 2015.

2. Ghavami A, Nutt MP and Hardy SP: Heat shock protein and high-dose aspirin: Effects on random skin flap survival in a rat model. Ann Plast Surg 48: 60-67, 2002.

3. Huemer GM, Froschauer SM, Pachinger T, Kwasny O and Schoffl H: A comparison of pretreatment with a topical combination of nonivamide and nicoboxil and surgical delay in a random pattern skin flap model. J Plast Reconstr Aesthet Surg 62: 914-919, 2009.

4. Li Q, Gao S, Yu Y, Wang W, Chen X, Wang R, Li T, Wang C, Li X and Wu X: A novel bFGF antagonist peptide inhibits breast cancer cell growth. Mol Med Rep 6: 210-214, 2012.

5. Zhang L, Mai HM, Zheng J, Zheng JW, Wang YA, Qin ZP and Li KL: Propranolol inhibits angiogenesis via down-regulating the expression of vascular endothelial growth factor in hemangioma derived stem cell. Int J Clin Exp Pathol 7: 48-55, 2014.

6. Cui K, Zhou X, Luo J, et al: Dual gene transfer of bFGF and PDGF in a single plasmid for the treatment of myocardial infarction. Exp Ther Med 7: 691-696, 2014.

7. Kim DS, Ko JH, Jeon YD, Han YH, Kim HJ, Poudel A, Jung HJ, Ku SK, Kim SJ, Park SH, et al: Ixeris dentata NAKAI Reduces Clinical Score and HIF-1 Expression in Experimental Colitis in Mice. Evid Based Complement Alternat Med 2013: 671281, 2013.

8. Koukourakis MI, Giatromanolaki A, Chong W, et al: Amifostine induces anaerobic metabolism and hypoxia-inducible factor 1 alpha. Cancer Chemother Pharmacol 53: 8-14, 2004.

9. Weidemann A, Klanke B, Wagner M, Volk T, Willam C, Wiesener MS, Eckardt KU and Warnecke C: Hypoxia, via stabilization of the hypoxia-inducible factor HIF-1alpha, is a direct and sufficient stimulus for brain-type natriuretic peptide induction. Biochem J 409: 233-242, 2008.

10. Lee SH, Lee JH, Yoo SY, Hur J, Kim HS and Kwon SM: Hypoxia inhibits cellular senescence to restore the therapeutic potential of old human endothelial progenitor cells via the hypoxia-inducible factor-1 $\alpha$-TWIST-p21 axis. Arterioscler Thromb Vasc Biol 33: 2407-2414, 2013.

11. Ren H, Lin D, Mou Z and Dong P: The adverse effect of selective cyclooxygenase-2 inhibitor on random skin flap survival in rats. PLoS One 8: e82802, 2013.

12. Nakamura M, Oda M, Inoue J, Ito T, Akiba Y, Kitajima M, Tsuchiya $\mathrm{M}$ and Ishii $\mathrm{H}$ : Plasticity of myofibroblasts appearing in granulation tissues after acetic acid treatment. Effect of bFGF. Dig Dis Sci 40: 2477-2480, 1995.

13. Wang TT, Yuan Y, Kang Y, Yuan WL, Zhang HT, Wu LY and Feng ZT: Effects of acupuncture on the expression of glial cell line-derived neurotrophic factor (GDNF) and basic fibroblast growth factor (FGF-2/bFGF) in the left sixth lumbar dorsal root ganglion following removal of adjacent dorsal root ganglia. Neurosci Lett 382: 236-241, 2005. 
14. Mohideen MA, Hruska-Hageman A and Nilsen-Hamilton M: A unique bFGF-responsive transcriptional element. Gene 237: 81-90, 1999.

15. Fayazzadeh E, Ahmadi SH, Rabbani S, Boroumand MA, Salavati A and Anvari MS: A comparative study of recombinant human basic fibroblast growth factor (bFGF) and erythropoietin (EPO) in prevention of skin flap ischemic necrosis in rats. Arch Iran Med 15: 553-556, 2012.

16. Wang F, Chang M, Shi Y, Jiang L, Zhao J, Hai L, Sharen G and Du H: Down-regulation of hypoxia-inducible factor-1 suppresses malignant biological behavior of triple-negative breast cancer cells. Int J Clin Exp Med 7: 3933-3940, 2014.

17. Nanduri J, Vaddi DR, Khan SA, Wang N, Makarenko V, Semenza GL and Prabhakar NR: HIF-1 $\alpha$ activation by intermittent hypoxia requires NADPH oxidase stimulation by xanthine oxidase. PLoS One 10: e0119762, 2015.

18. Koçer G, Nazıroğlu M, Çelik Ö, Önal L, Özçelik D, Koçer M and Sönmez TT: Basic fibroblast growth factor attenuates bisphosphonate-induced oxidative injury but decreases zinc and copper levels in oral epithelium of rat. Biol Trace Elem Res 153: 251-256, 2013.

19. Wang Z, Zhang H, Xu X, Shi H, Yu X, Wang X, Yan Y, Fu X, Hu H, Li X, et al: bFGF inhibits ER stress induced by ischemic oxidative injury via activation of the PI3K/Akt and ERK1/2 pathways. Toxicol Lett 212: 137-146, 2012.

20. Tasso R, Gaetani M, Molino E, Cattaneo A, Monticone M, Bachi A and Cancedda R: The role of bFGF on the ability of MSC to activate endogenous regenerative mechanisms in an ectopic bone formation model. Biomaterials 33: 2086-2096, 2012.

21. Rodriguez-Miguelez P, Lima-Cabello E, Martínez-Flórez S, Almar M, Cuevas MJ, González-Gallego J: Hypoxia-inducible factor-1 modulates the expression of vascular endothelial growth factor and endothelial nitric oxide synthase induced by eccentric exercise. J Appl Physiol (1985) 118: 1075-1083, 2015.
22. Liu L, Zhang J, Li M, Zhang X, Zhang J, Li Z, Wang L, Wu J and Luo C: Inhibition of HepG2 cell proliferation by ursolic acid and polysaccharides via the downregulation of cyclooxygenase-2. Mol Med Rep 9: 2505-2511, 2014.

23. Gallindo RM, Gonçalves FL, Figueira RL, Pereira LA, Simões AL, Schmidt AF and Sbragia L: Ventilation causes pulmonary vascular dilation and modulates the NOS and VEGF pathway on newborn rats with CDH. J Pediatr Surg 50: 842-848, 2015

24. Erdem H, Yildirim U, Uzunlar AK, Cam K, Tekin A, Kayikci MA, Sahiner C, Oktay M, Ankarali H and Aydin LY: Relationship among expression of basic-fibroblast growth factor, MTDH/astrocyte elevated gene-1, adenomatous polyposis coli, matrix metalloproteinase 9, and COX-2 markers with prognostic factors in prostate carcinomas. Niger J Clin Pract 16: 418-423, 2013.

25. Seo Y, Ji YW, Lee SM, Shim J, Noh H, Yeo A, Park C, Park MS, Chang EJ and Lee HKL Activation of HIF-1 $\alpha$ (hypoxia inducible factor- $1 \alpha$ ) prevents dry eye-induced acinar cell death in the lacrimal gland. Cell Death Dis 5: el309, 2014.

26. Hong JP, Li XM, Li MX and Zheng FL: VEGF suppresses epithelial-mesenchymal transition by inhibiting the expression of Smad3 and miR192, a Smad3-dependent microRNA. Int J Mol Med 31: 1436-1442, 2013.

27. Kranz A, Rau C, Kochs M and Waltenberger J: Elevation of vascular endothelial growth factor-A serum levels following acute myocardial infarction. Evidence for its origin and functional significance. J Mol Cell Cardiol 32: 65-72, 2000.

28. Chen T, Ren Z, Ye LC, Zhou PH, Xu JM, Shi Q, Yao LQ and Zhong YS: Factor inhibiting HIF1 $\alpha$ (FIH-1) functions as a tumor suppressor in human colorectal cancer by repressing HIF1 $\alpha$ pathway. Cancer Biol Ther 16: 244-252, 2015.

29. Tzeng HE, Chen PC, Lin KW, Lin CY, Tsai CH, Han SM, Teng CL, Hwang WL, Wang SW and Tang CH. Basic fibroblast growth factor induces VEGF expression in chondrosarcoma cells and subsequently promotes endothelial progenitor cells-primed angiogenesis. Clin Sci (Lond) 129: 147-158, 2015 